\title{
Sensitivity of Polarimetric Waveguide Interferometer for Different Wavelengths
}

\author{
K. Gut*, A. Zakrzewski and T. Pustelny \\ Department of Optoelectronics, Silesian University of Technology, Krzywoustego 2, 44-100 Gliwice, Poland \\ This publication presents experimental measurement methodology which allows to determine the character- \\ istics of sensitivity planar differential interferometer. To confirm data reliability additional measurements were \\ done such as simulations in OptiBPM software by Optiwave. Curves received from two methods: experimental \\ and theoretical, were summed up and compared. Conclusions were made on their basis, for example the influence \\ of used wavelengths or refractive index of single mode waveguide cladding on curves of sensitivity differential \\ interferometer using planar waveguide received from the ion exchange method.
}

PACS numbers: 42.25.Hz, 42.25.-p, 42.70.-a, 42.82.-m, 42.82.Et, 68.35.Ct

\section{Introduction}

Orthogonal $m$-order modes propagating in planar waveguide are different from themselves because of propagation constants $\beta_{\mathrm{TE}_{m}} \neq \beta_{\mathrm{TM}_{m}}$. Because of this, they propagate with different phase velocity. That is why, after going a way which is $z$-long between propagating modes $\mathrm{TE}_{m}$ and $\mathrm{TM}_{m}$, phase difference $\Delta \psi$ occurs, which is defined as [1]:

$$
\Delta \psi=\left(\beta_{\mathrm{TE}_{m}}-\beta_{\mathrm{TM}_{m}}\right) z,
$$

where $\Delta \psi$ - phase difference between the propagating modes, $\beta_{\mathrm{TE}_{m}}, \beta_{\mathrm{TM}_{m}}$ - TE and TM $m$-order mode propagation constants, $z$ - length of the path of propagation of the modes.

If the phase difference between the modes amounts to $2 \pi$ after passing path of propagation $L_{\mathrm{B}_{m}}$ it is called the beat length $m$-pair of orthogonal modes [1]:

$$
2 \pi=\left(\beta_{\mathrm{TE}_{m}}-\beta_{\mathrm{TM}_{m}}\right) L_{\mathrm{B}_{m}} \text {. }
$$

The functioning of most waveguide planar sensors is based on the differential interference phenomenon and detected by measuring the phase difference $\Delta \psi$ between propagating modes $\mathrm{TE}_{m}$ and $\mathrm{TM}_{m}$ at the end of the waveguide.

The basic element of these groups of waveguide sensors is the active layer put on the surface of the waveguide, which influenced by external factors changes their parameters [2]. The propagating modes in the waveguide are characterized by $m$-order propagation constants $\beta_{m}$ and the effective refractive index $N_{\mathrm{eff}_{m}}$. Because the value $N_{\mathrm{eff}_{m}}$ depends on the refractive index of the cladding [3], so changing the parameters of the active layer with the external factors we can directly influence the propagation

\footnotetext{
* corresponding author; e-mail: kgut@polsl.pl
}

constants of the modes, which results in changes of the measured phase difference $\Delta \psi$ between the propagating modes $\mathrm{TE}_{m}$ and $\mathrm{TM}_{m}$.

Waveguide interferometer sensors are characterized by the highest sensitivity, inaccessible for other sensors [4-11]. Such a high sensitivity is achieved thanks to the fact that in their rule of operation they base on the interference of light. To check the reliability of this common belief, this publication presents curves of planar differential interferometer sensitivity due to two methods: experimental and theoretical which includes simulations made in OptiBPM.

The sensitivity of a differential interferometer is defined as the derivative of difference of the effective refractive index of $m$-order modes to the refractive index of the cladding waveguide [12]:

$$
S=\frac{\mathrm{d}\left(N_{\mathrm{eff}\left(\mathrm{TE}_{m}\right)}-N_{\mathrm{eff}\left(\mathrm{TM}_{m}\right)}\right)}{\mathrm{d}\left(n_{\mathrm{c}}\right)},
$$

where $N_{\text {eff }}\left(\mathrm{TE}_{m}\right)-N_{\text {eff }}\left(\mathrm{TM}_{m}\right)$ - difference of the effective refractive index of TE and TM $m$-order modes, $n_{\mathrm{c}}$ refractive index of cladding waveguide.

\section{Measurement methodology}

Figure 1 shows the test stand for directly determining the difference of the propagation constants pair orthogonal modes, i.e. $\left(\mathrm{TE}_{0}\right.$ and $\left.\mathrm{TM}_{0}\right),\left(\mathrm{TE}_{1}\right.$ and $\left.\mathrm{TM}_{1}\right)$ etc. With respect to those waveguides, the influence of the refractive index of the cover on the differences of the modes' propagation constants was determined.

The system presented in Fig. 1 was used in the measurements $[12,13]$. The investigated planar waveguide was excited by means of a prism coupler PC. By adjusting of the polarization input beam appropriately, it was possible to excite the modes $\mathrm{TE}_{i}$ and $\mathrm{TM}_{i}$, simultane- 


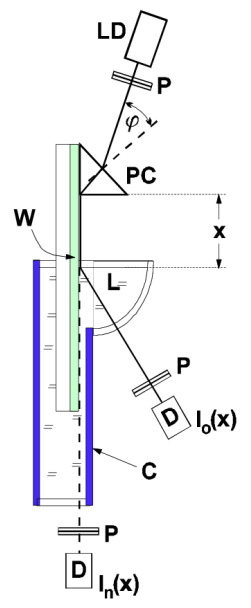

Fig. 1. Diagram of the measuring system: LD - laser diode, $\mathrm{PC}$ - prism coupler, $\mathrm{W}$ - waveguide structure, $\mathrm{P}$ - polarizer, $\mathrm{C}$ - container, $\mathrm{D}$ - detector, $\mathrm{L}$ immersion liquid [12].

ously. A definite pair of modes was selected by changing the angle of the beam input $\varphi$ to the prism.

When the refractive index of the immersion liquid $L$ is larger than the refractive index on the waveguide surface $n_{\mathrm{s}}$, then the difference between the propagation constants of both modes for $n_{\mathrm{c}}=1$ (air), derived from the recorded interference signal $I_{0}(x)$ is $\Delta \beta_{0}=2 \pi / \Delta x_{0}$ where $\Delta x_{0}$ the beat length defined as in Fig. 2. However, when we

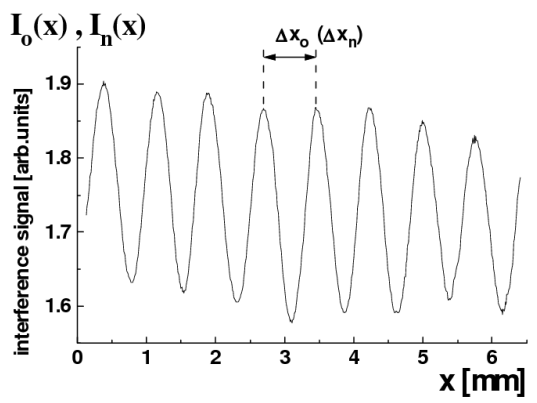

Fig. 2. Determination of the beat length $\Delta x_{0}$ and $\Delta x_{n}$ on the basis of the interference signal [12].

use the immersion liquid of the refractive index $n_{i}<n_{\mathrm{s}}$, we obtained the signal $I_{n}(x)$, and can determine difference $\Delta \beta_{i}=2 \pi\left(1 / \Delta x_{0}-1 / \Delta x_{n}\right)$, where $\Delta x_{n}$ - immersion of the waveguide in the liquid $n_{i}$ resulting in a change of the phase $\Delta \Phi=2 \pi$ at the waveguide output. In investigations involving the recording of the signal type $I_{0}(x)$, $\alpha$-bromonaphtalene $\left(n_{677}=1.6417\right)$ was used [12].

The values of the refractive indices of immersion liquids used in the measurements are given in Table. Using the technique described above, the measurements involving the differences of the propagation constants of ortogonal modes of the zero order were carried out for each investigated waveguide. The same immersion liquids were used
TABLE

Refractive indices of the applied immersion liquids.

\begin{tabular}{c|c|c}
\hline \hline Liquid & Refractive index & Wavelength [nm] \\
\hline cedar oil & 1.5004 & 677 \\
glycerin & 1.4664 & 677 \\
water + glycerin & 1.4490 & 589
\end{tabular}

in all measurements.

The measurements presented in Fig. 3a concern wavelengths of $664 \mathrm{~nm}, 650 \mathrm{~nm}, 532 \mathrm{~nm}$ and $405 \mathrm{~nm}$. The results received experimentally may be verified by comparing them with the theoretical results received. OptiBPM permits to determine the mode parameters which propagate inside the planar waveguide.

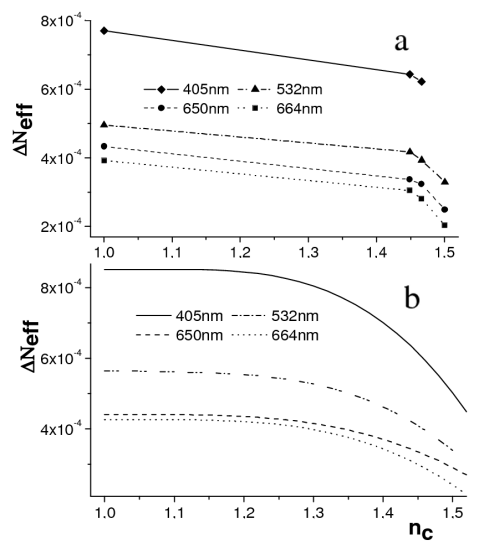

Fig. 3. (a) Dependence of the effective refractive index difference TE0 and TM0 on the refractive index of cladding. Curves received experimentally. (b) As in (a), but for zero-order modes. Curves received theoretically.

The process of planar waveguide modeling whose refractive profile was introduced in the diagram (Fig. 4) was implemented by adding other waveguide layers, describing its thickness and refractive index. Additionally, the refractive index of the cladding waveguide was defined, and took the same values as in experimental researches, at the beginning $n_{\mathrm{c}}=1$, then $1.4490,1.4664$, 1.5004 .

Simulations were performed for TE and TM polarizations concerning four wavelengths, the same as those used in experimental researches.

After each simulation we received a table with information: mode number, value of effective refractive index and kind of the polarization mode.

The results obtained by means of the Optiwave software displaying the dependence of the effective refractive index difference $\Delta N_{\text {eff }\left(\mathrm{TE}_{0}-\mathrm{TM}_{0}\right)}$ on the refractive index of cladding $n_{\mathrm{c}}$ for a single mode waveguide which is achieved in the ion-exchange process of $\mathrm{Na}^{+}-\mathrm{K}^{+}$on glass $\mathrm{BK} 7$, the process temperature was $400^{\circ} \mathrm{C}$, the process time $0.5 \mathrm{~h}$ can be seen in Fig. $3 \mathrm{~b}$. 


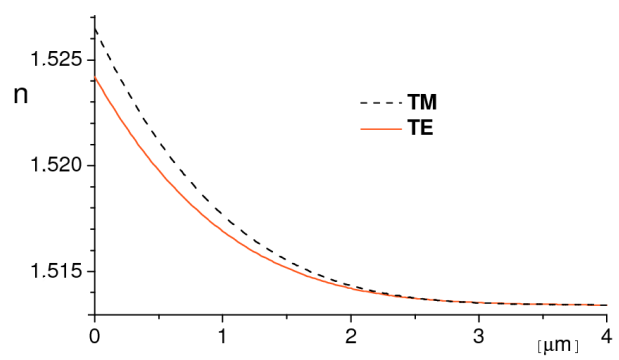

Fig. 4. Refractive single mode waveguide's investigated profile [14].

The conformability of the measured and numerically obtained data is quite good.

\section{Result and conclusions}

After all the simulations the effective refractive index was received, on the basis of which sensitivity curves on the planar differential interferometer for four used wavelengths were determined. The characteristics quoted below (Fig. 5) show the determined sensitivity for four applied wavelengths: $405 \mathrm{~nm}, 532 \mathrm{~nm}, 650 \mathrm{~nm}, 664 \mathrm{~nm}$.

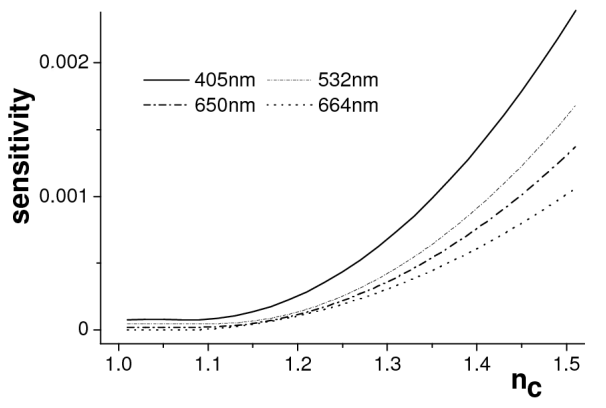

Fig. 5. Dependence of the sensitivity of the differential interferometer on the refractive index of cladding.

An interesting fact is that at higher values the refractive index of cladding $\left(n_{\mathrm{c}}>1.3\right)$, the sensitivity curves closely depended on the used wavelength; the higher the value of the parameter $\lambda$, the smaller is the maximum sensitivity level.

\section{Summary}

The paper presents measurements of the difference of the constants of propagation concerning various wavelengths in planar waveguides, obtained by ion-exchange in glass BK7. The data obtained by measurements coincide with those obtained numerically.

However, in the presented researches a single mode waveguide was used, but the test stand allows to research multi-mode waveguides, too. The test stand does not introduce any limitations for the usage of sources of light. Thanks to that, the sensitivity of the differential interferometer can be determined practically for every light beam with wavelength from the whole spectrum range. If we are only interested in measurements of the beat length $m$-pair orthogonal modes $L_{\mathrm{B}_{m}}$ versus wavelength function, then it is sufficient to use only the first stage of measurements. Knowing the value $L_{\mathrm{B}_{m}}$ it is possible to determine very important parameters, e.g. the difference of propagation constants or the effective refractive index of TE and TM $m$-order modes.

\section{Acknowledgments}

The work was sponsored by the State Committee for Scientific Research within the Structural Program WND-POIG.01.03.01-00-159/08-00.

\section{References}

[1] T. Pustelny, Physical and Technical Aspects of Optoelectronic Sensors, SUT University of Technology, Gliwice 2005.

[2] T. Tamir, Integrated Optics, Springer-Verlag, New York 1979

[3] W. Lukosz, Sensors Actuators B 29, 37 (1995).

[4] K. Gut, Acta Phys. Pol. A 114, 121 (2008).

[5] K. Gut, J. Phys. IV 137, 91 (2006).

[6] K. Gut, J. Phys. IV 129, 109 (2005).

[7] K. Gut, K. Nowak, Eur. Phys. J.-Spec. Top. 154, 89 (2008).

[8] M. Błahut, D. Kasprzak, Acta Phys. Pol. A 116, 257 (2009).

[9] M. Błahut, D. Kasprzak, M. Sujewicz, Acta Phys. Pol. A 116, 264 (2009).

[10] M. Błahut, K. Bazan, Acta Phys. Pol. A 116, 257 (2009).

[11] M. Błahut, D. Kasprzak, Opt. Appl. 33, 613 (2003).

[12] R. Rogoziński, K. Gut, P. Karasiński, Z. Opilski, A. Opilski, Proc. SPIE 3581, 375 (1998).

[13] K. Gut, A. Opilski Proc. SPIE 3744, 430 (1999).

[14] K. Gut, P. Karasiński, A. Opilski, Z. Opilski, in: Proc. 11th Europ. Conf. on Solid-State Transducer, Ed. W. Sohler, University of Munster, Munster 1997, p. 1547. 